\title{
Norois
}

Environnement, aménagement, société

$216 \mid 2010 / 3$

Territoire, Paysage, Anthropisation, Perception,

Conservation, Restauration

\section{Les pêches maritimes en Afrique centrale : les préalables à une exploitation durable des} ressources

Sea fishings in Central Africa: prerequisites in a sustainable exploitation of the resources

\section{Guy-Serge Bignoumba}

\section{(2) OpenEdition}

Journals

Édition électronique

URL : https://journals.openedition.org/norois/3341

DOI : $10.4000 /$ norois.3341

ISBN : 978-2-7535-1564-2

ISSN : $1760-8546$

Éditeur

Presses universitaires de Rennes

Édition imprimée

Date de publication : 15 novembre 2010

Pagination : 47-56

ISBN : 978-2-7535-1257-3

ISSN : 0029-182X

\section{Référence électronique}

Guy-Serge Bignoumba, "Les pêches maritimes en Afrique centrale : les préalables à une exploitation durable des ressources », Norois [En ligne], 216 | 2010/3, mis en ligne le 01 décembre 2012, consulté le 13 janvier 2022. URL : http://journals.openedition.org/norois/3341 ; DOI : https://doi.org/10.4000/ norois.3341 


\title{
LES PÊCHES MARitimes en Afrique CENTRALE : LES PRÉALABLES À UNE EXPLOITATION DURABLE DES RESSOURCES
}

\author{
Guy-Serge Bignoumba \\ (Université Omar-Bongo), \\ BP 7655 LibreVILLE - Gabon \\ gsbignoumba@yahoo.fr
}

\section{RÉSUMÉ}

Au regard du faible volume des captures, de la déficience de l'outil de production, du sous-équipement des infrastructures portuaires et d'un marché de commercialisation étriqué, la pêche maritime en Afrique centrale apparaît comme une activité peu développée, tant en pêche industrielle qu'en pêche artisanale. Mais les avantages divers qu'elle est censée procurer, notamment en termes de création d'emplois, de distribution de revenus ou d'apport alimentaire, en font un des piliers majeurs de la politique économique et sociale dans la sous-région. Pour y parvenir effectivement, la politique des pêches doit s'inscrire dans une perspective de développement durable, fondée sur une bonne connaissance du potentiel exploitable, une préservation des ressources et leur appropriation par les capacités nationales.

MOTS CLÉ : Afrique centrale - pêche maritime - pêche industrielle - pêche artisanale - développement durable - potentiel exploitable - préservation des ressources - maind'œuvre stable - pêche illicite

\section{ABSTRACT \\ Sea fishings in Central Africa: prerequisites in a sustainable exploitation of the resources}

Given the low catching volume, the underequipment of past infrastructures, the deficiencies of the production tool and a narrow trade market, sea fishing in Central Africa seems to be an undeveloped activity both in industrial and small scale fishing. But the various benefits it is likely to bring, especially in terms of job creation, income distribution or food supply, make of sea fishing one of the pillars of the economic and social policy of the sub region. But, to achieve this goal, the fishing policy must lie within the framework of sustainable development, based notably on a good knowledge of the exploitable potential, the preservation of resources and their appropriation by national capacities.

KEY WORDS : Central Africa - sea fishing - large scale fishing - small scale fishing fishing policy - sustainable development - exploitable potential - preservation of resources - stable labour force - illicit fishing 
Sur la base des données statistiques tirées de la FAO, il apparaît qu'en 2006, la production mondiale des pêches maritimes est évaluée à 82000000 de tonnes selon les statistiques de la FAO. L'Asie occupe de loin la première place, avec des captures évaluées à 41200000 tonnes. Elle est suivie de l'Amérique et de l'Europe, avec respectivement 22000000 et 13000000 tonnes.

L'Afrique vient en quatrième position avec 4500000 tonnes, soit une production inférieure à celle d'un pays comme l'Indonésie (4700000 millions de tonnes). Le Maroc en détient le leadership avec 865000 tonnes. Il est suivi de l'Afrique du Sud (617000 tonnes) et du Nigeria (552000 tonnes), ce qui place dans le peloton de tête, un pays d'Afrique du Nord, un d'Afrique Australe et un autre d'Afrique de l'Ouest. Il faut attendre la dixième place pour voir apparaître un pays d'Afrique centrale (figure 1), en l'occurrence le Congo Démocratique, avec 237000 tonnes.

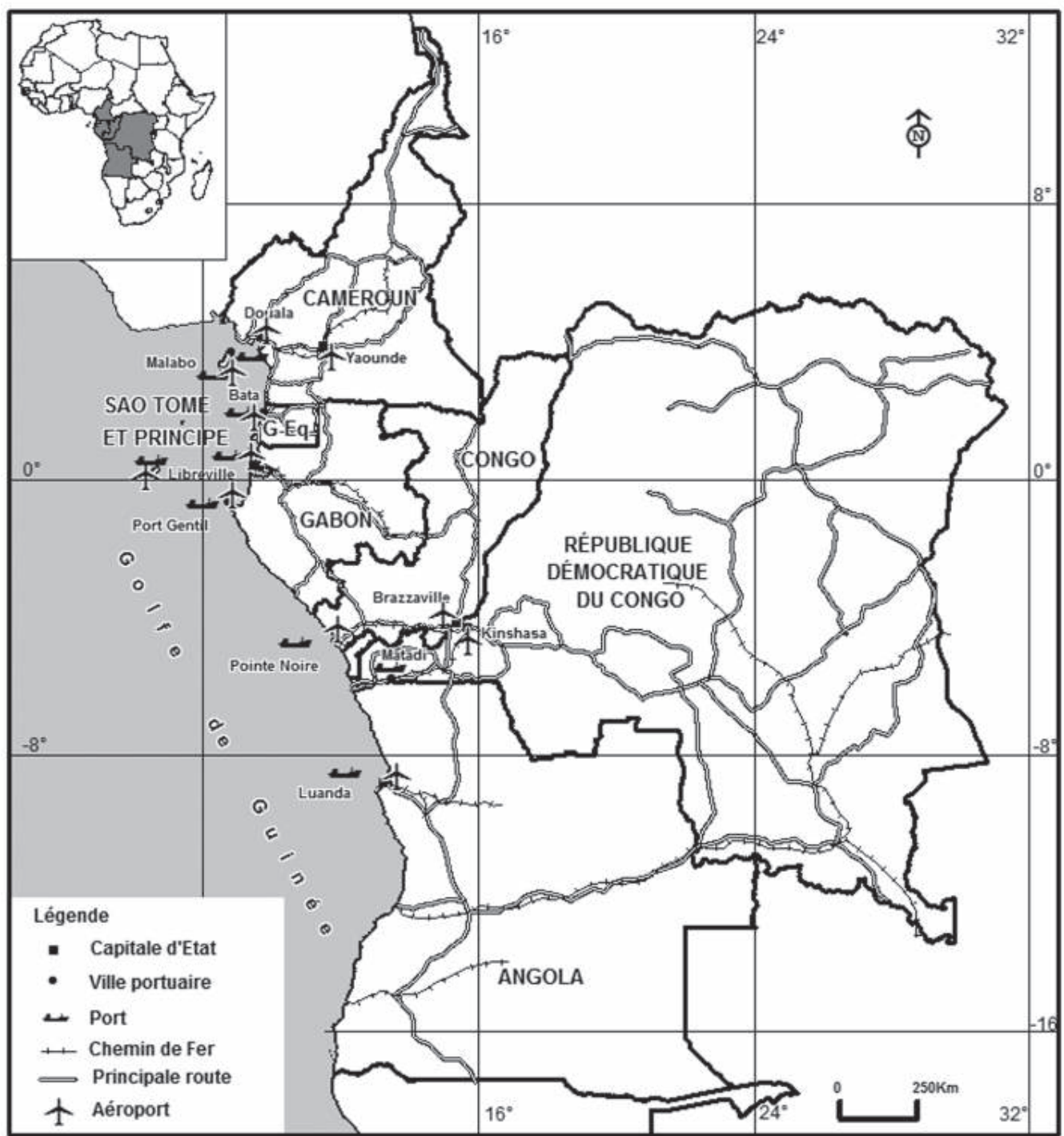

Figure 1 : L'afrique centrale maritime

Maritime central Africa 
Ce classement ${ }^{1}$, qui traduit les faibles performances de la pêche maritime en Afrique centrale, est en contradiction avec l'ambition des États de faire de cette activité, un des secteurs de promotion économique et sociale depuis qu'elle a été classée au nombre des cinq piliers retenus dans le cadre du programme économique régional 2009-2015, pour l'Afrique centrale (Perdrix et Dougueli, 2009). Mais toute politique des pêches se voulant efficace doit viser le développement durable, et s'assurer que les conditions propres à atteindre cet objectif sont réunies. Aussi, après un état des lieux succinct des pêches maritimes dans la sous-région, au travers de l'analyse de la structure spatiale suggérée par l'activité halieutique, l'article va-t-il s'articuler autour de la nécessité d'un développement durable des pêches maritimes en Afrique centrale, qui repose principalement sur une bonne connaissance des ressources, une préservation de celles-ci et une main-d'œuvre stable.

\section{Un espace halieutique peu développé}

Il existe une grande diversité disciplinaire autour des problématiques halieutiques, où chaque discipline se distingue par la spécificité de son objet d'étude.

C'est ainsi par exemple que l'économie se préoccupera des coûts occasionnés par l'exploitation des armements de pêche, la sociologie s'intéressera notamment aux acteurs du secteur et à leur origine sociale, le droit s'attachera à l'étude du cadre réglementaire, etc. Quant à la géographie, elle privilégiera l'approche spatiale, par l'analyse de la structure spatiale produite par l'activité de pêche. Autrement dit, elle étudiera l'espace halieutique, qui s'articule autour d'un triptyque (Corlay, 1979, 1997), c'est-à-dire d'une construction trivalente et intégrée, constituée, en amont, d'un espace de capture difficilement identifiable; au centre, d'un espace portuaire plus ou moins aménagé et en aval, d'un espace de commercialisation aux dimensions variables. Ce triptyque servira de fil conducteur à l'aperçu des pêches maritimes en Afrique centrale dont les traits caractéristiques sont notamment un faible volume des débarquements, doublé d'une stabilité des prises au cours des dernières années.

\section{DES PÊCHERIES MARQUÉES PAR À UN FAIBLE NIVEAU DES CAPTURES}

Les pêcheries maritimes d'Afrique centrale se caractérisent par un faible niveau de captures et une tendance à une légère augmentation des prises. Seule l'Angola connaît une évolution en dents de scie de sa production entre 2000 et 2007 (fig. 2 - planche IV). On distingue quatre groupes de pays, à savoir l'Angola et la République Démocratique du Congo, dont la production oscille entre 146000 et 255000 tonnes; le Gabon et le Congo (38000-59000 tonnes); la Guinée Équatoriale et Sao-Tomé et Principe (2700-7000 tonnes). Le Cameroun fait figure de singleton avec une production oscillant entre 102000 et 142000 tonnes.

Les captures enregistrées en pêche artisanale sont toujours supérieures à celles de la pêche industrielle, quel que soit le pays ${ }^{2}$. Cette situation est le fait d'un plus grand nombre de pêcheurs et d'une flottille, essentiellement piroguière ${ }^{3}$, plus fournie dans un secteur artisanal où les investissements sont moindres que dans le secteur industriel. Le Tarif Extérieur Commun (TEC) de la Communauté Économique et Monétaire d'Afrique centrale très élevé pour le matériel de pêche et les navires (20\% auxquels s'ajoute une TVA de plus de $18 \%$ ), et le coût des intrants, notam-

1. Pour 2006, le classement, par ordre décroissant, est le suivant: Maroc, Afrique du Sud, Nigeria, Namibie, Sénégal, Égypte, Ouganda, Ghana, Tanzanie, Congo Démocratique, Angola.

2. À titre d'exemple, sur les 28480 tonnes produites par le Congo en 2006, 17980 ont été fournies par la pêche artisanale et 10500 par la pêche industrielle (www. congo.relais-infocom.net). Au Cameroun, sur les 70000 tonnes produites en 2007 dans la pêche maritime, 10000 l'ont été par le secteur industriel et 60000 par celui de l'artisanat [www.afriquejet.com].

3. En 2005, le Congo comptait un total de 1347 pirogues pour 10 armements et 29 navires de pêche industrielle basés à Pointe-Noire [www.congo.relais-infocom.net]. Il y a donc un avantage numérique des pirogues sur les navires modernes, ce qui est le cas dans tous les pays. Au Gabon, l'enquête cadre des pêcheries maritimes artisanales (DGPA, 2009), réalisée en 2009, a comptabilisé 1190 pêcheurs et 763 pirogues dont 150 sans moteur. Dans le secteur industriel, on dénombre 39 bateaux de pêche, non compris la flottille de l’Union Européenne, ni celle du Japon (DGPA, s.d.). 
ment du carburant qui peut atteindre jusqu'à $40 \%$ du chiffres d'affaires au Gabon, sont autant de contraintes qui ne favorisent guère l'investissement dans le secteur industriel (BAD, www.afdb.org).

Les deux secteurs souffrent de sérieuses déficiences et du vieillissement ${ }^{4}$ de leurs outils de production ainsi que de la quasi-absence d'un système de transformation industrielle des captures. Ils pâtissent également du sous-équipement de leurs infrastructures portuaires.

\section{LE SOUS-ÉQUIPEMENT PORTUAIRE}

L'espace portuaire peut revêtir un caractère sommaire, comme dans le cas des échouages qui s'égrènent le long des littoraux des pays sub-sahariens. Mais cet espace peut aussi prendre la forme d'un aménagement des plus évolués, notamment dans les pays développés, qui ont atteint un certain niveau « d'industrialisation » de leur activité de pêche. En Afrique centrale, la plupart des ports de pêche artisanale n'ont pas dépassé le stade de l'échouage, un type d'aménagement qui s'accommode fort bien de l'usage de pirogues, souvent monoxyles, affranchies de tout équipement lourd et qui symbolisent le mieux la pêche artisanale en Afrique. Quant aux ports de pêche industrielle, ils se distinguent par leur sous-équipement et par les difficultés dues à la faible profondeur de leurs plans d'eau.

La bathymétrie joue un rôle essentiel dans le développement de toute activité halieutique, par l'influence qu'elle exerce sur les mouvements des navires dans un port. Par exemple, au port môle de Libreville, port de pêche de la capitale, les navires d'un tirant d'eau supérieur à trois mètres ne peuvent accoster ou quitter le port qu'à marée haute, ce qui impacte considérablement le volume des débarquements. Au port de Sao-Tomé, on déplore également la faible profondeur des eaux qui empêche la réception des bateaux de fort tonnage et qui nécessite le recours aux barges pour récupérer les cargaisons au loin. Par ailleurs, le sous-équipement du port, notamment en termes d'outils de transbordement et d'espaces de stockage, agit sur la qualité des produits, du fait des multiples manipulations qu'ils subissent, ou sur leurs conditions de conservation en chambres froides. Ici, se pose le problème de la rareté ou même de l'inexistence de ces équipements, ce qui justifie la présence de l'homme dans les opérations de transbordement des produits. On relève également une faible capacité de stockage des chambres froides, dont un grand nombre est consacré à des activités sans lien direct avec la pêche (Bignoumba, 2000). Une autre difficulté rencontrée par ces pays est leur forte dépendance vis-à-vis de l'extérieur pour ce qui est du marché des produits de pêche.

\section{UNE FORTE DÉPENDANCE À L'ÉGARD DES MARCHÉS EXTÉRIEURS}

Le rôle du marché de consommation n'est plus à démontrer dans sa capacité à impulser le développement de toute activité économique. Cette capacité est d'autant plus déterminante que l'activité concernée s'insère dans une dynamique marchande visant le profit maximal grâce aux facilités d'écoulement offertes. Le marché doit être apprécié dans sa dimension quantitative, c'està-dire par le nombre de ses consommateurs potentiels, lequel dépend de la taille de la population dans chaque pays. Mais il doit être également vu sous son angle qualitatif, c'est-à-dire par rapport au pouvoir d'achat des populations, autrement dit de leur capacité à consommer (tableau 1).

Le marché de consommation en Afrique centrale souffre de sa taille réduite et de sa dépendance vis-à-vis de l'extérieur.

Si la première est tributaire de la faible démographie de l'Afrique centrale, pour la seconde, la sousrégion paye aussi de son incapacité à réaliser l'intégration de son système économique, qui condamne les États à compter d'abord sur un marché national souvent étriqué, comme c'est le cas pour le Gabon, la Guinée Équatoriale ou Sao-Tomé et Principe. En réalité, il faut insérer les contraintes de

4. Il est fait allusion ici aux navires de pêche industrielle, souvent en faible nombre, et souvent constitués d'unités de secondemain en raison des difficultés des armateurs à acquérir des bateaux neufs, faute de financements. 


\begin{tabular}{|l|c|c|}
\hline \multicolumn{1}{|c|}{ Pays } & Population (en millions) & Consommation (Kg par hab.) \\
\hline Angola & 19 & 13,7 \\
\hline Cameroun & 20 & 15,0 \\
\hline Centrafrique & 4,5 & 4,1 \\
\hline Tchad & 11,5 & 6,1 \\
\hline RDC & 67,8 & 5,4 \\
\hline Congo & 3,8 & 20,4 \\
\hline Guinée Equatoriale & 0,7 & 27,2 \\
\hline Gabon & 1,5 & 40,4 \\
\hline Sao-Tomé\&Principe & 0,17 & 24,5 \\
\hline
\end{tabular}

Tableau 1 : Population et consommation de poisson en Afrique centrale (Source : ONU, 2009 et FAO, 2006) Population and consumption of fish in Central Africa

l'Afrique centrale dans un contexte continental déjà fort peu favorable, et à propos duquel A.-V. Hoh et B. Vinaux (2006), citant le Programme des Nations Unies pour le Développement (PNUD), soulignent que « dans la compétition mondiale, l'Afrique subsaharienne se caractérise par l'étroitesse de son marché, avec un produit intérieur brut (PIB) de quelque 248 milliards d'euros, inférieur à celui de l'Australie. La moitié des habitants n'y disposent que d'un dollar par jour. ». R. Pourtier (2003) précise au demeurant que l'Afrique centrale est effectivement une région sous-peuplée et confrontée à des problèmes d'insertion économique et sociale difficiles à résoudre partout.

Aux contraintes démographiques, s'ajoutent les problèmes structurels, notamment ceux liés aux liaisons difficiles entre les différents pays, qui ne favorisent guère les échanges inter-États; d'où la place tenue par l'extérieur dans l'écoulement des produits et leur approvisionnement (tableau 2).

\begin{tabular}{|l|c|c|}
\hline \multicolumn{1}{|c|}{ Régions } & Importations & Exportations \\
\hline Afrique de l'Ouest & 58,1 & 0,0 \\
\hline Amérique du Sud & 15,3 & 0,0 \\
\hline Afrique du Nord & 7,6 & 0,1 \\
\hline Chine & 4,8 & 9,1 \\
\hline Union Européenne & 3,6 & 88,4 \\
\hline Afrique centrale & 0,4 & 2,0 \\
\hline Asie du Sud/Est & 0,6 & 0,4 \\
\hline
\end{tabular}

Tableau 2 : Part des importations et exportations en 2004-2006 (en \% de la valeur totale des pêches) (Source : FAO, appendice III - direction du commerce extérieur, 2006)

Share of imports and exports in 2004-2006 (as \% of total value of fisheries)

De fait, l'Afrique centrale n'occupe que la $11^{\mathrm{e}}$ place dans les importations de la sous-région, là où l'Afrique de l'Ouest, suivie de l'Amérique du Sud, en détient le leadership. Elle occupe en revanche une bien meilleure position dans les exportations grâce à sa 3e place derrière l'Union Européenne et la Chine.

Les exportations concernent principalement des produits à forte valeur marchande, comme les crevettes du Cameroun, de la République du Congo ou du Gabon, vendues à l'Union Européenne, et secondairement des produits moins rémunérateurs tels que la sardine fumée écoulée en Afrique de l'Ouest où ce produit reste fortement apprécié ${ }^{5}$.

5. La sardine donne lieu à un fort trafic, notamment entre des pays d'Afrique centrale et ceux d'Afrique de l'Ouest. Mais le caractère le plus souvent informel de cette activité ne permet de maîtriser ni les flux réels ni les réseaux ainsi développés. 
Au sortir de cet état des lieux, il apparaît plus que nécessaire de développer les pêches maritimes en Afrique centrale; ce qui permettrait non seulement d'accroître les captures, mais également d'augmenter les revenus des opérateurs et de contribuer à la réduction de la pauvreté. La pêche pourrait ainsi participer à l'essor social et économique de la sous-région. Pour ce faire, les politiques à mettre en œuvre doivent s'inscrire dans la durée, et viser une exploitation durable des ressources, qui exige au minimum une bonne connaissance du potentiel exploitable, une préservation des ressources et l'implication d'une main-d'œuvre stable.

\section{Les conditons d'une pêche durable en Afrique centrale}

Sur la base du diagnostic fait plus haut, l'essor des pêches maritimes en Afrique centrale appelle, entre autres, l'aménagement des infrastructures portuaires, le renforcement de l'appui institutionnel aux pêcheurs par les facilités d'accès aux financements en vue de l'acquisition des outils de production, l'encadrement technique des opérateurs, etc. Mais au-delà de ce qui apparaît classique, tout projet de développement des pêches en Afrique centrale se voulant durable doit répondre au moins à trois exigences : une bonne connaissance des ressources, une stratégie de conservation de celles-ci et leur appropriation par des capacités nationales ou à tout le moins par des communautés de pêcheurs stabilisées et peu enclines à la migration perpétuelle.

\section{LA CONNAISSANCE DES RESSOURCES}

La réussite des plans d'aménagement des pêcheries maritimes réside dans la disponibilité de données fiables et actualisées sur l'état des ressources. Il est en effet difficile de concevoir une stratégie d'aménagement rationnelle des pêcheries lorsque l'on ignore le potentiel biologique exploitable sur lequel vont s'appliquer les mesures de gestion, car l'effort de pêche doit être ajusté à la taille du stock. Or en Afrique centrale, les données disponibles remontent souvent à plusieurs années et sont tirées des campagnes d'évaluation menées avec l'appui financier et technique de la coopération internationale. Ainsi en est-il par exemple de l'intervention dans la sous-région, du navire océanographique norvégien Fridtjof Nansen au cours des années 1980 et 1990, ou de celle plus récente (début des années 2000) de scientifiques espagnols au Gabon pour une évaluation des ressources. Ces campagnes sont aussi le fait d'organismes spécialisés du système des Nations Unies tels que la FAO ou le fruit d'accords bilatéraux comme au Gabon.

Toutefois, le fait de voir certaines campagnes réalisées par des pays tiers exploitant ces mêmes ressources soulève le problème de la sincérité des résultats de ces évaluations, tant en ce qui concerne la taille du stock, la composition des espèces que leur localisation. Dès lors se pose la question fondamentale de savoir jusqu'où un Etat évaluateur des ressources, et candidat à l'exploitation de ces mêmes ressources, est prêt à aller sans courir le risque de compromettre ses propres intérêts.

Compte tenu de la rareté, sinon de l'inexistence dans la plupart des pays, d'une expertise nationale en matière d'évaluation de stocks, le moyen le plus avisé d'y aboutir consisterait dans l'organisation de ces campagnes d'évaluation dans un cadre communautaire, au travers notamment des activités de la COREP ${ }^{6}$. Cette voie offrirait l'avantage d'une économie des ressources financières par la mutualisation des moyens, en même temps qu'elle permettrait une meilleure vision de l'étendue de stocks qui, parfois, couvrent une aire transcendant les limites territoriales des États, pour rechercher la cohérence qu'autorise la constitution d'unités biologiques homogènes. De la sorte, il serait plus aisé d'envisager une exploitation concertée des ressources, vers laquelle à terme l'Afrique centrale est contrainte de s'acheminer, au nom d'une harmonisation des politiques de mise en valeur des ressources halieutiques, notamment des espèces pélagiques qui ignorent les frontières des États.

6. Commission Régionale des Pêches du Golfe de Guinée, devenue depuis peu un organe spécialisé de la CEEAC (Communauté Économique des États de l'Afrique centrale) en charge des questions des pêches dans la sous-région. 
Depuis plusieurs années, le Gabon procède annuellement aux fermetures temporaires de certaines pêcheries, crevettières et sardinières, pour nécessité de repos biologique. Si l'utilité de ces mesures ne fait guère de doute, l'on ne peut manquer de s'interroger sur les données objectives qui sous-tendent ces décisions administratives; les principales questions étant posées sur la pertinence des périodes choisies, les secteurs mis en fermeture, les espèces à préserver, etc. Autant de questions que seule une connaissance aussi précise que possible des stocks et de la dynamique des populations permettrait d'y répondre.

\section{LA PRÉSERVATION DES RESSOURCES}

L'exploitation durable des ressources halieutiques ne peut être sérieusement envisagée sans une politique de préservation de celles-ci. Il faut entendre par là, une utilisation précautionneuse des ressources, faisant en sorte que les générations futures puissent en jouir, au moins dans les mêmes proportions que les générations actuelles; ce qui appelle des mesures préventives et répressives.

Les premières mesures portent sur une sensibilisation des communautés de pêcheurs aux principes d'une exploitation responsable des ressources. Dans leur acception la plus simple, elles visent à faire adhérer les pêcheurs au respect des dispositions réglementaires en matière de pêche que bien souvent ignorent les pêcheurs, faute souvent d'un déficit d'information de la part de l'administration. Ces mesures visent également l'implication des pêcheurs dans le processus de gestion des ressources, notamment dans le cadre de comités de co-gestion, au sein desquels ils sont associés à l'État dans la conception, l'élaboration et l'application des principes de bonne gestion des ressources. C'est du fait de leur mise à l'écart dans le processus de mise en œuvre de ces mesures de gestion que certains pêcheurs en viennent à tourner le dos aux décisions dont ils n’appréhendent pas toujours le bien fondé.

Les secondes mesures portent sur l'organisation d'un système de surveillance côtière visant à s'assurer du respect de la réglementation en matière de pêche, depuis la fréquentation des lieux de capture jusqu'à l'usage des engins et techniques de pêche. En effet, des cas sont légion où des navires de pêche industrielle viennent pêcher trop près des côtes, dans des zones pourtant réservées à la pêche artisanale, causant au passage de sérieux dégâts sur la faune et la flore aquatiques. La surveillance constitue également l'un des moyens les plus éprouvés de lutte contre le braconnage des ressources par des navires non nationaux.

Cette opération fort coûteuse nécessite un personnel bien formé et du matériel performant inexistant dans la sous-région, la surveillance côtière reste ainsi mal assurée dans la plupart des États de l'Afrique centrale. C'est ainsi par exemple que le Gabon a installé au sein de la Direction Générale des Pêches et de l'Aquaculture, un système de surveillance par satellite de ses pêcheries industrielles, où les bateaux, munis de balises à leur bord, peuvent être suivis à la trace. Toutefois, ce système, que vient d'adopter le Cameroun, reste pour l'heure limité à quelques navires nationaux tout en échappant encore aux bateaux de pêche des pays tiers qui exploitent les eaux gabonaises, et sera renforcé par un projet interministériel de plus grande envergure. Faisant suite à une étude de faisabilité réalisée par un cabinet israélien, ce projet, conduit par le Ministère en charge des Pêches, intègre notamment les Ministères chargés de la Défense Nationale et de la Marine Marchande. Il convient par ailleurs de noter les postes d'observation à terre installés par le WWF (World Wildlife Fund) le long des côtes gabonaises, notamment au large de Sette Cama. Munies de vedettes, les équipes de cette $\mathrm{ONG}$ internationale vont jusquà intercepter en mer des bateaux se livrant à une pêche illicite.

\section{L'APPROPRIATION DES RESSOURCES PAR LES CAPACITÉS NATIONALES}

Le faible niveau de développement des pêches maritimes en Afrique centrale prend racine, entre autres, dans un contexte culturel peu favorable, où la valeur des ressources humaines, aussi bien en quantité qu'en qualité reste insuffisante. Il faut dire que les ressources humaines 
locales font cruellement défaut dans la presque totalité des pays d'Afrique centrale. Partout, le secteur reste largement dominé par des pêcheurs non nationaux, dont la plupart sont originaires d'Afrique de l'Ouest. Au Cameroun, les statistiques du ministère de l'Élevage, des Pêches et des Industries Animales (MINEPIA) indiquent que sur 25000 pêcheurs côtiers, moins de 5000 sont Camerounais (APM Afrique ${ }^{7}$ ).

Les pêcheurs migrants forment des groupes ethno-professionnels mouvants et instables. Leurs séjours dans les pays d'accueil ne constituent souvent qu'une étape transitoire sur le chemin d'horizons plus prometteurs, bien que beaucoup s'y soient établis durablement ${ }^{8}$. Même si l'apport des pêcheurs migrants à la production halieutique des pays d'accueil demeure importante, il convient néanmoins de considérer que la politique des pêches dans ces pays ne saurait s'affranchir d'une appropriation des ressources par les capacités nationales; ce qui suppose une présence plus significative des pêcheurs locaux dans le secteur. L'efficacité des politiques de pêche initiées par l'administration doit s'appuyer sur des communautés stables, sur lesquelles l'État peut investir sur le long terme en capitalisant les acquis, toute chose difficile à réaliser lorsque les groupes ciblés sont mouvants et se renouvellent continuellement. Les effets positifs de la pêche, notamment en termes de création d'emplois, d'accès aux revenus et de lutte contre la pauvreté, devraient aider à l'entrée dans le secteur d'un plus grand nombre de pêcheurs nationaux ${ }^{9}$.

Au-delà de disposer de pêcheurs locaux plus nombreux, l'appropriation des ressources s'entend également des capacités des États à contrôler l'activité des navires étrangers dans leurs eaux. Ceci concerne notamment la pêche chinoise, et surtout celle pratiquée par des pays de l'Union Européenne, qui bénéficient d'accords de pêche prévoyant l'embarquement d'observateurs nationaux à bord des navires de pêche. Mais cette disposition n'a presque jamais été respectée, notamment au Gabon où l'administration évoque l'inexistence d'agents formés à l'observation des activités de pêche en même temps que l'impossibilité des thoniers européens à accoster au port môle de Libreville faute de fonds suffisants! Une solution à ce problème consisterait par exemple à user de la contrepartie financière versée à l'État pour payer la formation des agents. Sur l'accostage des navires, les thoniers pourraient très bien mouiller en rade, les observateurs se faisant transporter jusqu'aux bateaux par de plus petites embarcations. Dans tous les cas, l'absence de contrôle à bord des navires pose la question de la sincérité des déclarations de captures fournies par les navires non nationaux et suscite des doutes sur leur volume réel ainsi que sur les retombées financières qui s'y attachent.

\section{Conclusion}

Les conditions évoquées ici ne sont pas une panacée, et ne pourront pas seules, assurer une exploitation durable des ressources halieutiques en Afrique centrale. Cependant, elles n'en constituent pas moins les piliers, au milieu d'une batterie de mesures administratives et techniques à prendre pour permettre à la pêche de relever ses principaux défis, à savoir une contribution à l’indépendance alimentaire et à la lutte contre la pauvreté par la création d'emplois.

Toutefois, la politique des pêches en Afrique centrale doit répondre à une démarche intégrée, qui passe par l'harmonisation des politiques nationales en la matière. Les organes d'intégration communautaire que sont la COREP, la CEBEVIRHA (Commission économique du Bétail, de la Viande et des Ressources Halieutiques) ou la COMHAFAT (Conférence ministérielle sur la Coopération entre les États Africains Riverains de l'Océan Atlantique) ne doivent pas être des institutions de plus dans un paysage communautaire déjà abondamment administré. Ils se doivent d'agir sur le terrain par des propositions concrètes, stratégiques et concertées, notamment dans

\footnotetext{
7. Réseau « Agricultures Paysannes et Modernisation en Afrique ».

8. Au Gabon, certaines familles de pêcheurs migrants s'y sont installées depuis le XIXe siècle (Roux, 1950).

9. Un des enjeux du PSPA (Projet d'appui au Secteur des Pêches et de l'Aquaculture), co-financé par la Banque Africaine de Développement et le Gouvernement Gabonais est de former sur entre 2005 et 2010, 1500 pêcheurs dont 500 nouveaux.
} 
le sens d'une prévention des conflits dans le secteur des pêches. Ceux qui opposent depuis des années, les marins pêcheurs artisans gabonais et équato-guinénens de la Baie de la Mondah aux administrations du pays voisin pourraient être évités si des accords inter-étatiques prévoyaient par exemple le déplacement sans restriction des pêcheurs sur l'ensemble des pêcheries communautaires.

En fait, se pose ici le dilemme auquel sont confrontés les États d'Afrique centrale, de savoir comment concilier les impératifs de souveraineté nationale et la nécessité d'une intégration sous régionale dans une perspective de développement intégrée et communautaire de l'activité halieutique. L'enjeu est de taille car il s'agit d'éviter tout conflit d'intérêt entre les États, par la recherche des points de convergence que seule permet une approche systémique de l'espace halieutique sous régional, à laquelle la géographie pourrait avantageusement contribuer.

\section{Bibliographie}

APM AFRIQUE. État des lieux des organisations de pêche maritime et continentale en Afrique centrale, rapport de synthèse (cas du Cameroun, du Congo, du Tchad et du Gabon), Yaoundé, Secrétariat Permanent, 15 p. AFP, (2006), Pêche illégale : traquer les pirates, L’Union (quotidien), nº 9060, Libreville (non paginé).

Bignoumba G.-S., 2000. Anthropisation et conflits d'usage sur le littoral du Gabon : éléments de réflexion, Les Cahiers Nantais, n 53, p. 107-114.

Chauveau J.-P., 1991. Les variations spatiales et temporelles de l'environnement socio-économique et l'évolution de la pêche maritime artisanale sur les côtes onest-africaines. Essai d'analyse en longue période $\mathrm{XV}^{e}-\mathrm{XX} \mathrm{X}^{\mathrm{e}}$ siècle, Pêcheries ouest-africaines : variabilité, instabilité et changement, Paris, Ouest Éditions, p. 14-25.

Chaussade J., 1994. La mer nourricière : enjeu du XXI siècle, Champtoceaux, Imprimerie de la Concorde, $153 \mathrm{p}$.

Carré F., 1998. La pêche sur les côtes de l'Afrique tropicale, du Sénégal au Congo, dans Gamblin A. (dir.), Les littoraux espaces de vies, Paris, Sedes, coll. « DIEM », p. 297-306.

Corlay J.-P., 1979. La notion d'espace de production halieutique : proposition méthodologique d'étude à partir de l'exemple danois, Norois, $n^{\circ} 104$, p. 449-466.

Corlay J.-P., (éd.), 1997. Littoral 95, Actes du colloque « Continuités et ruptures sur les littoraux européens ", Cahiers Nantais, n 47-48, 512 p.

Delauney K., 1989. Approche historique de l'expansion des pêcheurs ghanéens (Fante et Ewe) sur le littoral ivoirien, Actes du colloque "La recherche face à la pêche artisanale », Montpellier, ORSTOM-IFREMER, p. 1005-1018

Djama Th., Nna Aвo'o, 1999. Aperçu de la pêche camerounaise, Cours ACP-UE sur la gestion des pêches et de la biodiversité, Dakar, 5 p. [http://www.fishbase.org.ph/].

Direction générale des PêChes et de l’Aquaculture, 2010. Données statistiques sur la taille et le type de bateaux ainsi que des captures en pêche industrielle au Gabon, inédit, 9 p.

Direction générale des Pêches et de l’Aquaculture, 2009. Rapport enquête cadre dans les pêcheries artisanales maritimes et continentales $d u$ Gabon, Libreville, République Gabonaise, 69 p.

Ekouala L., 2006. L'activité piscicole dans la province de l'Estuaire, Mémoire de Maîtrise de Géographie, Univ. Omar Bongo, Libreville, 140 p.

FAO, 2002. La République du Congo : données d'économie générale, FID/CP/PRC, Rev. 5, Rome, 8 p. [http// www fao.org/].

FAO, 2006. Annuaire statistique des pêches : tableaux récapitulatifs, Rome, non paginé [http//www.fao.org/].

Grenier J.-C., 2003. Pêche, halte au gaspillage, Écofinance, Le magazine des économies émergentes, $\mathrm{n}^{\circ}$ 37, p. $70-77$.

Hoh A.-V., Vignaux B., 2006. L’Afrique n'est plus l'eldorado des entreprises françaises, Le Monde Diplomatique, février 2006, p. 12-13.

Ministère de la Planification et de la Programmation du Développement (Gabon), sans date. Document de stratégie de croissance et de réduction de la pauvreté : synthèse et matrices, sl., 151 p. 
Guy-Serge Bignoumba

Perdrix Ph., Dougueli G., 2009. Où va l'Afrique centrale?, Jeune Afrique, nº 2518, p. 24-30.

Pourtier R., 2003. L'Afrique centrale et les régions transfrontalières : perspectives de reconstruction et d'intégration, OCDE, Initiative Afrique centrale, s.l., 76 p.

Roux C., 1950. Les principaux engins de pêche et les pêcheries des côtes de l'AEF, Actes du Congrès des pêches et des pêcheries dans l'Union Française Outre-Mer, Marseille, Institut Colonial de Marseille, p. 192-195.

Cet article a été reçu le 30 juillet 2008 et définitivement accepté le 30 janvier 2010. 
planche IV (Guy-Serge BIGNOUMBA - Les pêches maritimes en Afrique centrale...)

(Émilie BOURGET, Laurence LE DÛ-BLAYO - Définition d'unités paysagères par télédétection...)

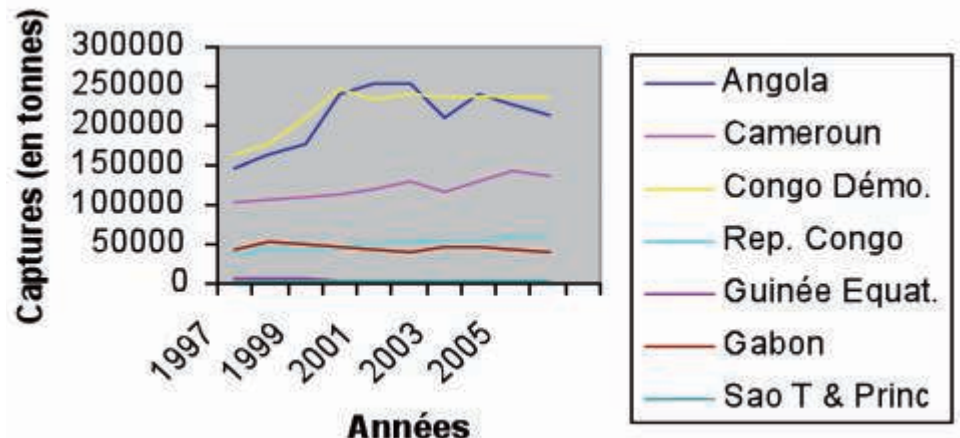

Figure 2 : Évolution des captures en Afrique centrale, de 1997 à 2006 (Source : FAO, captures par pays ou zones, 2006)

Evolution of captures in Central Africa, from 1997 till 2006

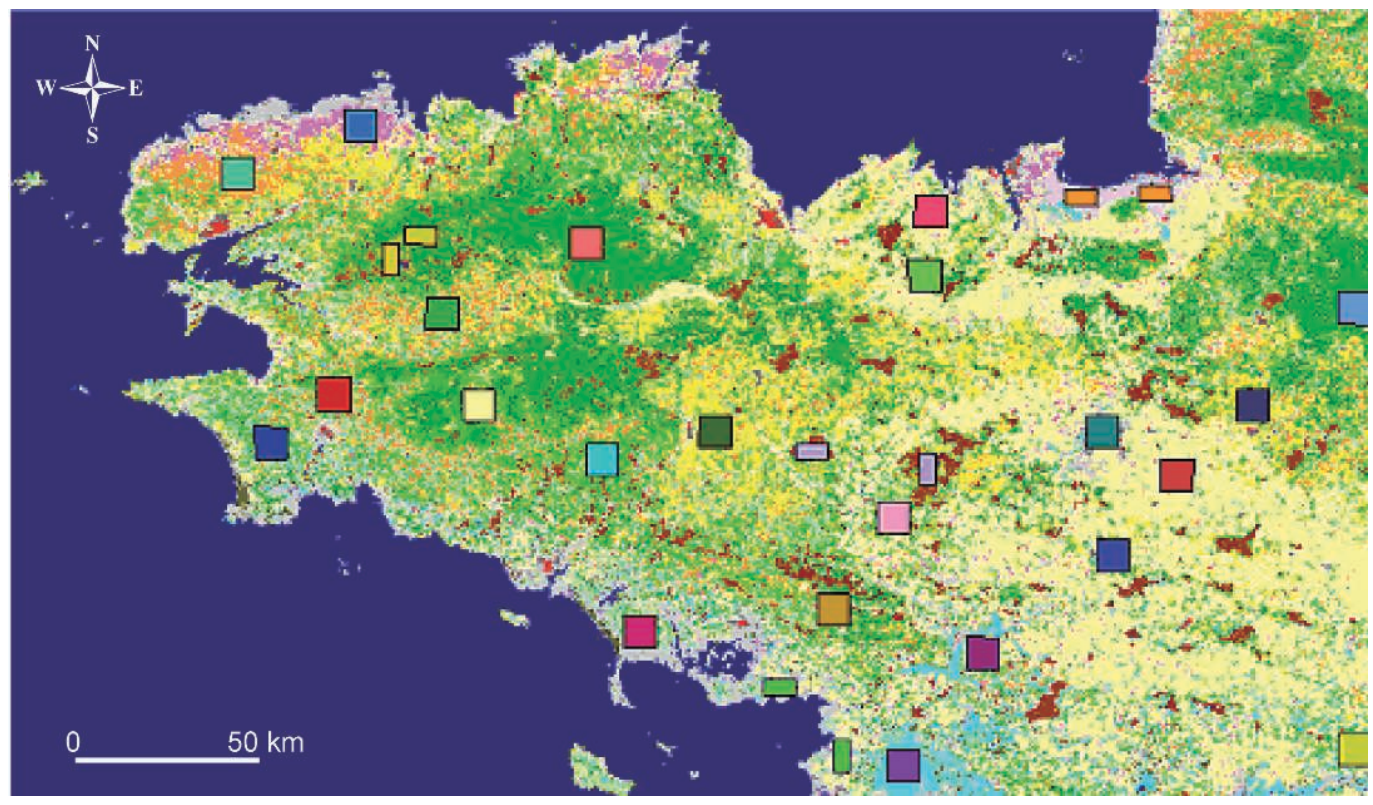

Figure 2 : Localisation des 26 zones de référence pour l'utilisation de ClaPaS (Bourget dans Le Dû-Blayo et al., $2008 \mathrm{~b})$

Location map of the 26 reference areas in ClaPaS using (Bourget in Le Dî-Blayo et al., 2008b) 